\title{
Ectopic expression of CXCL13, BAFF, APRIL and LT- $\beta$ is associated with artery tertiary lymphoid organs in giant cell arteritis
}

\author{
Francesco Ciccia, ${ }^{1}$ Aroldo Rizzo, ${ }^{2}$ Rosario Maugeri, ${ }^{3}$ Riccardo Alessandro, ${ }^{4}$ \\ Stefania Croci, ${ }^{5}$ Giuliana Guggino, ${ }^{1}$ Alberto Cavazza, ${ }^{6}$ Stefania Raimondo, ${ }^{4}$ \\ Alessandra Cannizzaro, ${ }^{2}$ Domenico Gerardo lacopino, ${ }^{3}$ Carlo Salvarani, $^{7}$ \\ Giovanni Triolo ${ }^{1}$
}

\begin{abstract}
Handling editor Tore K Kvien
- Additional material is published online only. To view please visit the journal online (http://dx.doi.org/10.1136/ annrheumdis-2016-209217).

For numbered affiliations see end of article.
\end{abstract}

\section{Correspondence to} Professor Giovanni Triolo, Dipartimento Biomedico di Medicina Interna e

Specialistica, Sezione di

Reumatologia, Piazza delle

Cliniche 2, 90127 Palermo,

Italy; giovanni.triolo@unipa.it

Received 18 January 2016 Revised 29 March 2016 Accepted 31 March 2016
To cite: Ciccia F, Rizzo A, Maugeri $\mathrm{R}$, et al. Ann Rheum Dis Published Online First: [please include Day Month Year] doi:10.1136/ annrheumdis-2016-209217

\section{ABSTRACT}

Objectives To investigate whether artery tertiary lymphoid organs (ATLOs) are present in giant cell arteritis (GCA) and that their formation is associated with the ectopic expression of constitutive lymphoid tissue-homing chemokines.

Methods Reverse transcriptase PCR, immunohistochemical and immunofluorescence analysis were used to determine the presence of ectopic ATLOs in GCA and the expression of chemokines/chemokine receptors and cytokines involved in lymphoneogenesis in the temporal artery samples obtained from 50 patients with GCA and 30 controls. The presence of lymphatic conduits, of follicular dendritic cells (FDCs) precursors and lymphoid tissue inducer cells was also investigated. Finally, expression of CXCL13, B cell activating factor (BAFF), a proliferation-inducing ligand (APRIL) and CCL21 by isolated myofibroblasts was evaluated before and after stimulation with Toll-like receptors (TLRs) agonists and cytokines.

Results ATLOs were observed in the media layer of $60 \%$ of patients with GCA in close proximity to high endothelial venules and independently by the age of patients and the presence of atherosclerosis. ATLO formation was also accompanied by the expression of CXCL13, BAFF, a proliferation-inducing ligand (APRIL), lymphotoxin (LT)- $\beta$, interleukin (IL)-17 and IL-7, the presence of FDC precursors and of lymphoid conduits. Stimulation of myofibroblasts with TLR agonists and cytokines resulted in the upregulation of BAFF and CXCL13.

Conclusions ATLOs occur in the inflamed arteries of patients with GCA possibly representing the immune sites where immune responses towards unknown arterial wall-derived antigens may be organised.

\section{INTRODUCTION}

Arteries are immunoprivileged sites. ${ }^{1}$ In advanced atherosclerotic lesions, however, adventitial lymphoid infiltrates, sometimes aggregated in lymphoid follicles (the so-called artery tertiary lymphoid organs (ATLOs)), occur together with marked neoangiogenesis and lymphangiogenesis, and with the extensive induction of high endothelial venules (HEVs). ${ }^{2}{ }^{3}$ The evidence that ATLO formation increases with age ${ }^{3}$ may suggest a role of these structures in disrupting the immune privilege of normal human arteries. Tertiary lymphoid organs (TLOs) are often observed also in autoimmune diseases, ${ }^{4}$ and their induction seems to be dependent on the aberrant expression of CXCL13, interleukin (IL)-7 and IL-17.

Giant cell arteritis (GCA), the prototype of large vessel vasculitis, is a chronic inflammatory disease occurring in the elderly, ${ }^{8}$ whose pathogenesis is still elusive. Dendritic cells residing in the vessel wall, in close proximity to vasa vasorum, seem to be important in initiating the pathogenic cascade by recruiting $\mathrm{T}$ cells and macrophages to form granulomatous infiltrates. ${ }^{9}$ B cells have been described mainly in the adventitia of GCA arteries, ${ }^{10}$ and recent evidences indicate a disturbed distribution of IL-6-producing B cells in GCA, suggesting a potential role of $\mathrm{B}$ cell compartment in the pathogenesis of the disease. ${ }^{11}$

Although adventitial ectopic lymphoid follicles have been demonstrated in chronic periaortitis, ${ }^{12}$ they have been never demonstrated in patients with GCA and the mechanisms underlying ATLOs formation in inflamed vessels not adequately studied. In this study, we studied the occurrence of ATLO structures and the expression of cytokines and chemokines involved in lymphoneogenesis in patients with GCA.

\section{PATIENTS AND METHODS}

\section{Patients}

Fifty patients with GCA (38 females, mean duration of disease $2 \pm 11$ months) who fulfilled the American College of Rheumatology classification criteria $^{13}$ and with biopsy-proven GCA were included in this study. At the time of temporal artery biopsy, all the patients were untreated and prior or current diagnosis of cancer, autoimmune disease other than GCA or chronic infection was carefully excluded. Their median age was 69 years (range 57-82 years) and erythrocyte sedimentation rate (ESR) was $62 \mathrm{~mm} / \mathrm{h}$ (range 35-108). Headache and artery tenderness and/or decreased or absent temporal artery pulsation were present in 30 out of 50 patients. The baseline inflammatory response was also assessed as described by HernándezRodríguez et al. ${ }^{14}$ Briefly, four parameters (score range 0-4) were evaluated at diagnosis: fever, weight loss, an ESR of $85 \mathrm{~mm} / \mathrm{h}$ and a haemoglobin 
level of $11.0 \mathrm{gm} / \mathrm{dL}$. Histologically, normal temporal artery samples from 30 consecutive patients (20 women, 10 men; median 71 years, range 63-86 years) with suspected GCA but with negative biopsy results were considered as controls.

\section{Immunohistochemical analysis of GCA biopsy samples}

Immunohistochemistry was performed on 5 - $\mu$ m-thick paraffin-embedded sections from temporal artery biopsies (TABs) and from tonsils (used as positive controls) as previously described. ${ }^{15}$ Ten sequential sections were obtained and artery biopsy specimens were evaluated by two experienced pathologists (AR and $\mathrm{AC}$ ) with an expertise in vasculitides with no access to clinical data. The intra-rater agreement and the interrater agreement calculated by the Cohen's $\mathrm{K}$ coefficient for the two pathologists were 0.84 and 0.79 , respectively. The cellular infiltrates and the presence of ATLOs were assessed on the basis of the histological appearance and confirmed by immunohistochemical staining of sequential sections with monoclonal antibodies to CD3, CD20, CD21 and PNAd (MECA-79) (see online supplementary table $\mathrm{S} 1$ ) in order to evaluate the presence of follicular dendritic cells (FDCs) networks, as previously described. ${ }^{16}$ Temporal arteries were classified either as without ATLOs (when no clear compartmentalisation of $\mathrm{T}$ and $\mathrm{B}$ cells could be recognised) or with ATLOs (when inflammatory aggregates displayed a well-defined organisation in separated T-cell-rich and B-cell-rich areas). Expression of lymphoid chemokines such as CXCL13, lymphotoxin (LT) $-\beta$ and B cell activating factor (BAFF) regulating the recirculation of lymphocytes and dendritic cells to and within lymphoid structures is one criterion that defines TLO. ${ }^{4}$ For this reason, staining for CXCL13, lymphotoxin (LT)- $\beta$ and BAFF was performed on sections sequential to those used to evaluate the presence of ATLOs. In order to study atherosclerosis, the sections were stained with Masson"s trichrome (Sigma-Aldrich). Immunohistochemistry was performed on 5 - $\mu \mathrm{m}$-thick paraffin-embedded sections from temporal arteries and from tonsils used as positive controls as previously described. ${ }^{15} \mathrm{~A}$ list of primary antibodies is provided in online supplementary table S1. The primary antibodies were added and incubated for $1 \mathrm{~h}$ at room temperature. Isotype-matched irrelevant antibodies were used as a negative control. Following three washes with Tris buffered saline, slides were incubated for $30 \mathrm{~min}$ with peroxidase-conjugated Dako EnVision polymer. After three further washings, peroxidase activity was visualised using diaminobenzidine chromogen (Dako), and slides were lightly counterstained with haematoxylin before dehydration and mounting in DePex (VWR

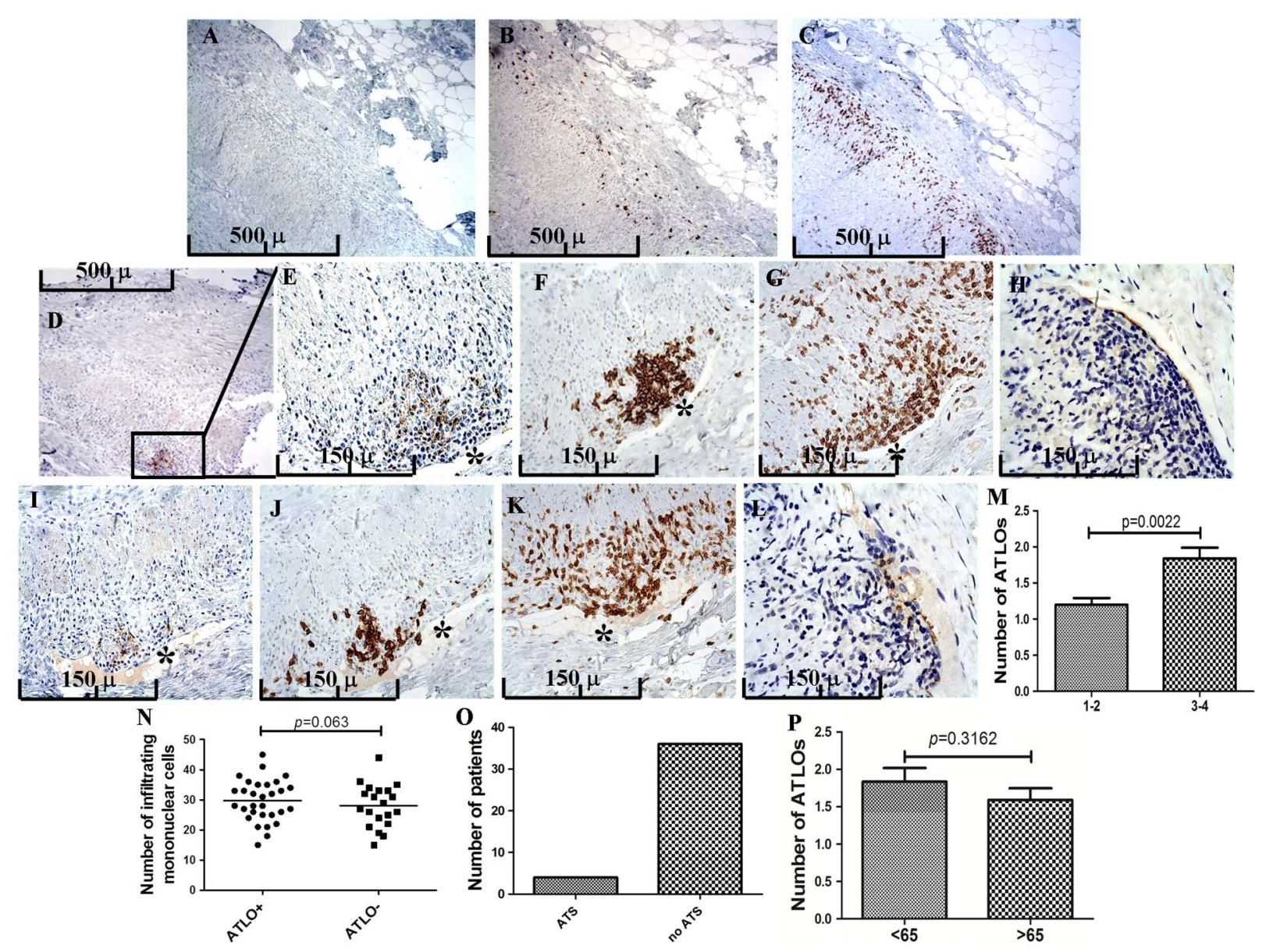

Figure 1 Artery tertiary lymphoid organ (ATLO) structures in temporal arteries of giant cell arteritis (GCA). Representative images showing the distribution of CD21 (A), CD19 (B) and CD3 (C) positive cells in temporal arteries without ATLOs. Representative images showing CD21 (D-E), CD19 $(F)$ and CD3 (G) positive cells in GCA temporal arteries with ATLOs. (H) Representative image showing PNAd+ high endothelial venules at the edge of ATLOs structures. Representative images showing CD21 (I), CD19 (J) and CD3 (K) positive cells in GCA arteries with ATLOs. (L) Representative image showing PNAd+ high endothelial venules at the edge of ATLOs structures. (M) Higher number of ATLOs were observed in patients with GCA with higher systemic inflammation. ATLOs were observed independently by the number of infiltrating mononuclear cells (N), the presence of atherosclerosis $(0)$ and/or with the patient's age $(P)$. $(A-D)$ Original magnification $\times 100$; $(E-L)$ original magnification $\times 250$. Data are expressed as mean (SEM). ATS, atherosclerosis. 
International, Oslo, Norway). The number of immunoreactive cells was determined by counting positively stained cells on photomicrographs obtained from three random high-power microscopic fields (400 $\times$ magnification) under a Leica DM2000 optical microscope using a Leica DFC320 digital camera (Leica, Rijswijk, The Netherlands). To characterise FDCs, perivascular precursor sections were incubated with unlabelled antihuman NG2, CXCL12 and platelet-derived growth factor receptors (PDGFR)- $\beta$ and then treated with Fluorescein isothiocyanate (FITC)-conjugated or rhodamine red-conjugated antirabbit or antimouse antibodies plus RNasi $(200 \mathrm{mg} / \mathrm{mL})$ and counterstained using Toto-3 iodide (642/660; Invitrogen). Confocal analysis was used to acquire fluorescence staining.

\section{Primary cultures of myofibroblasts from human temporal arteries}

Primary cultures of myofibroblasts from TABs were obtained at the Arcispedale Santa Maria Nuova-IRCCS, Reggio Emilia (Italy) following the method developed by Lozano et al. ${ }^{17}$ Briefly, TABs were cut in $1 \mathrm{~mm}$ sections, placed in $25 \mu \mathrm{L}$ matrigel drops (BD Biosciences, San Jose, California, USA) and covered with Dulbecco's modified Eagle medium (DMEM) with high glucose (Euroclone, Italy) $+10 \%$ fetal bovine serum (FBS), $2 \mathrm{mM}$ glutamine and $50 \mu \mathrm{g} / \mathrm{mL}$ gentamicin. Primary cultures obtained from TABs showed a myofibroblast phenotype because they expressed smooth muscle alpha actin and fibroblast activating protein but did not express or expressed at low levels von Willebrand factor evaluated by real-time PCR. Primary cultures were seeded at a density of 40000 cells per well in six-well plates in $2 \mathrm{~mL}$ DMEM with high glucose $+10 \%$ fetal bovine serum (FBS), $2 \mathrm{mM}$ glutamine and $50 \mu \mathrm{g} / \mathrm{mL}$ gentamicin. After $24 \mathrm{~h}$, they were treated with IL-1 $\beta(10 \mathrm{ng} / \mathrm{mL})$, IL-6 $(10 \mathrm{ng} / \mathrm{mL})$, interferon (IFN) $\gamma(10 \mathrm{ng} / \mathrm{mL})$ (premium grade from Miltenyi Biotec), IL-17 $(50 \mathrm{ng} / \mathrm{mL})$ (research grade from Miltenyi Biotec), PolyIC (10 $\mu \mathrm{g} / \mathrm{mL})$ and lipopolysaccharide (LPS, $100 \mathrm{ng} /$ $\mathrm{mL}$ ) (from Sigma) in $2 \mathrm{~mL}$ DMEM with high glucose $+10 \%$ fetal bovine serum (FBS), $2 \mathrm{mM}$ glutamine and $50 \mu \mathrm{g} / \mathrm{mL}$ gentamicin. Cells were collected after 6 and $24 \mathrm{~h}$ of treatment, total RNA extracted with the miRNeasy micro kit (QIAGEN, Milan, Italy) and $62.5 \mathrm{ng}$ RNA retro-transcribed with the Takara reverse transcription kit in a total volume of $20 \mu \mathrm{L}$ following the manufacturer's instruction.

\section{RNA extraction from artery biopsies and quantitative TaqMan reverse transcriptase PCR}

TABs were also stored in RNAlater solution (Applied Biosystems, Foster City, California, USA). Reverse transcriptase PCR (RT-PCR) was performed as previously described. ${ }^{15}$ Master mix and TaqMan gene expression assays for Glyceraldehyde 3-phosphate dehydrogenase (GAPDH) control and target genes were obtained from Applied Biosystems (Foster City). A list of primers is provided in online supplementary table S2.

\section{Statistical analysis}

Statistical analysis of quantitative variables was performed using the Mann-Whitney rank-sum test. Pearson's correlation analysis was used to quantify the expression associations between the genes of interest. $\mathrm{p}$ Values $<0.05$ were considered significant.

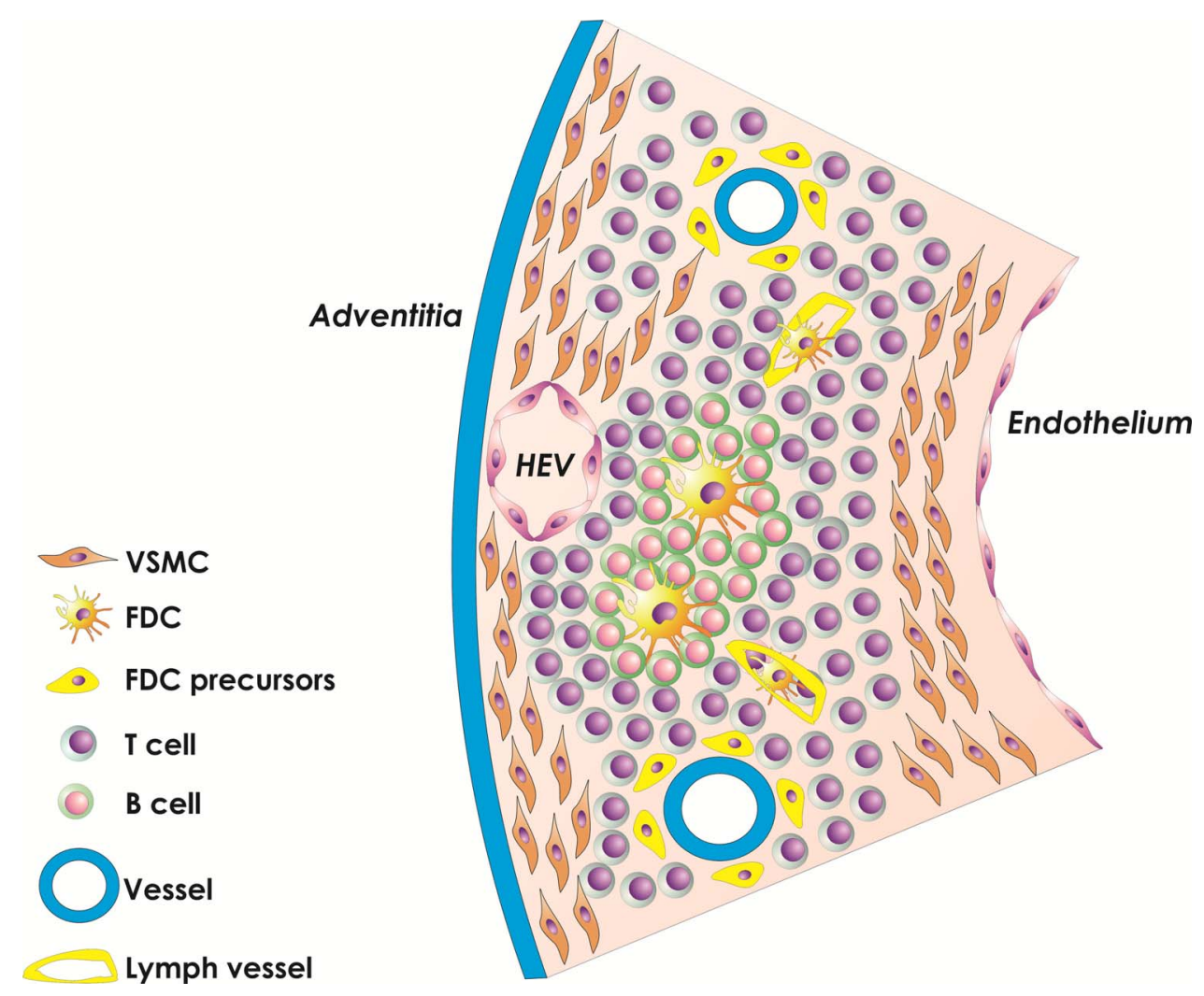

Figure 2 Artery tertiary lymphoid organs (ATLOs) are present in the media layer of giant cell arteries. ATLO structures are organised in separate T cell areas, B cell follicles and a network of follicular dendritic cells (FDCs). FDC precursors are present in close proximity of vasa vasorum and may participate in the formation of ATLO structures. Newly formed lymphatic conduits may serve to transport chemokines and antigens. HEV, high endothelial venules. 


\section{RESULTS}

ATLOs are present in GCA temporal arteries and correlate with systemic inflammatory responses

All the patients enrolled had a biopsy-proven GCA with temporal transmural inflammation. In order to detect ATLOs, sequential temporal artery samples from patients and controls were stained with anti-CD3, anti-CD20 and anti-CD21 antibodies. Evidence of diffuse T cells infiltration without the concomitant B cells presence was observed in 20 of 50 patients with GCA (figure $1 \mathrm{~A}-\mathrm{C}$ ). In the remaining 30 patients with GCA, B cells were invariably associated with a defined T cell/B cell segregation surrounding a central area consisting of $\mathrm{CD} 21^{+}$ cells (FDCs) (figure 1D-G,I-K). Twenty-five of these arteries $(83 \%)$ displayed only one area of T/B/FDC compartmentalisation, the remaining five displaying up to two areas of $\mathrm{T} / \mathrm{B} / \mathrm{FDC}$ compartmentalisation. ATLOs were never observed in control temporal arteries. ATLOs were invariably observed near to vascular structure morphological resembling HEVs (figures $1 \mathrm{E}-\mathrm{G}$,
I-L asterisks and 2) exclusively located in the context of media layer. We next evaluated whether such vessels express PNAd, the addressin system typical of secondary lymphoid organs. As shown in figures $1 \mathrm{H}, \mathrm{L}$ and $2, \mathrm{PNAd}^{+}$HEVs were observed at the edge of lymphoid aggregates, in all the patients with GCA displaying ATLOs. The number of ATLOs was significantly correlated with parameters of systemic inflammatory response (figure 1M) but not with duration of symptoms prior to biopsy and specific GCA features such as jaw claudication, headache, scalp tenderness and permanent visual loss (data not shown). We also investigated whether the degree of arterial inflammation, evaluated by counting infiltrating inflammatory mononuclear cells, the presence of giant cells and intimal disruption, was the main factor associated with ATLOs. No significant differences in the numbers of infiltrating inflammatory cells, presence of giant cells and intimal disruption between nonsegregated versus segregated arteries (figure $1 \mathrm{~N}$ ) were found, indicating that the number of inflammatory cells alone and the
A

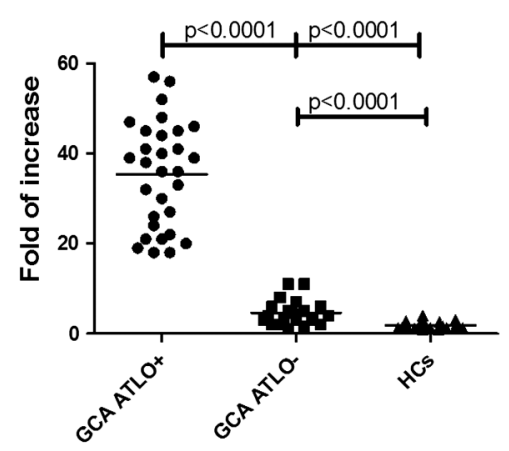

D

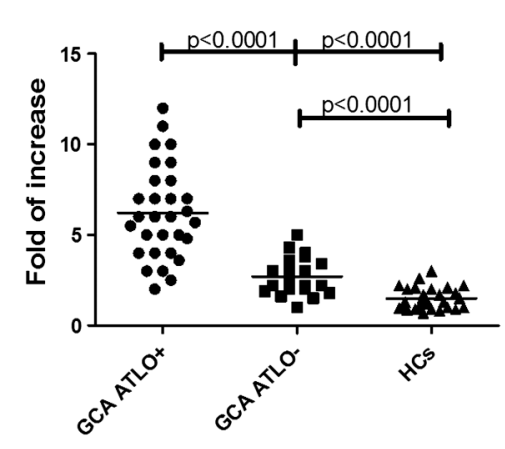

B

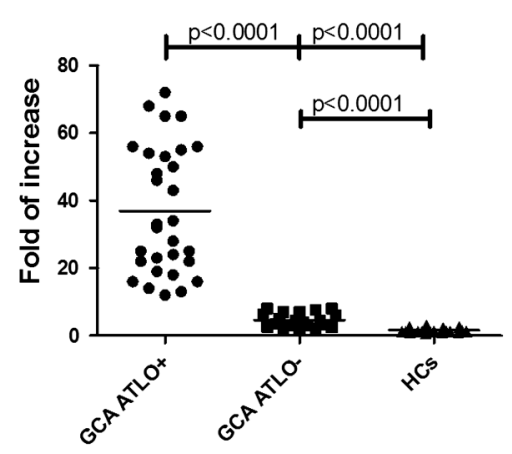

E

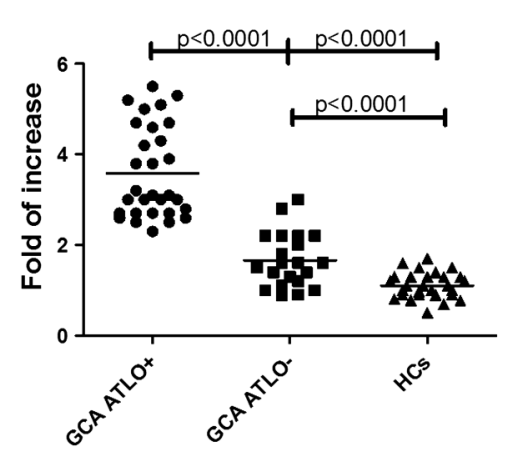

C

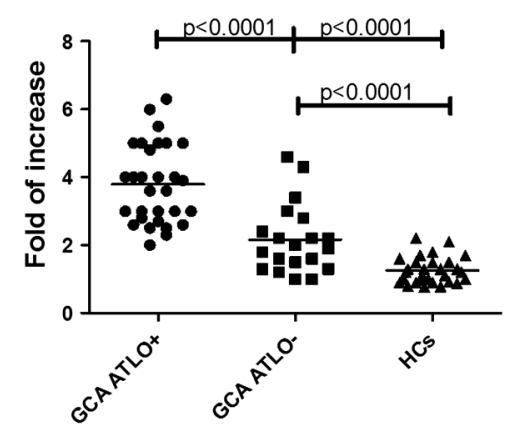

F

IL-7

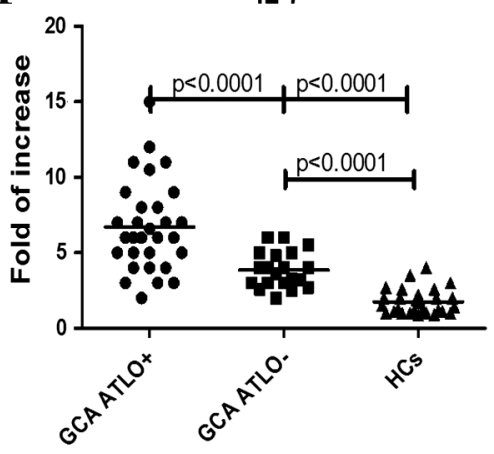

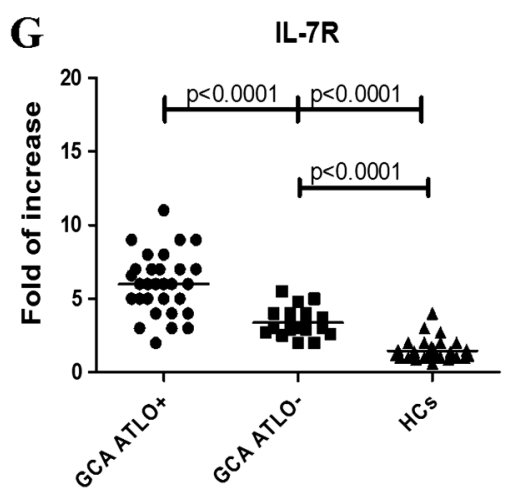

H

IL-17

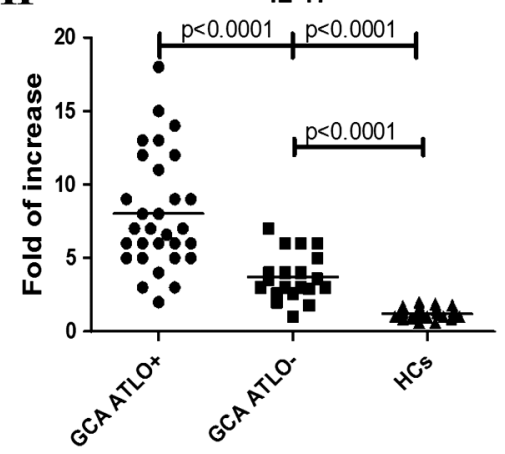

Figure 3 Chemokine expression in giant cell arteritis (GCA) temporal arteries. Relative mRNA levels of CXCL13 (A), CXCR5 (B), lymphotoxin (LT)- $\beta$ (C), B cell activating factor (BAFF) (D), a proliferation-inducing ligand (APRIL) (E), interleukin (IL)-7 (F), IL-7R (G) and IL-17 (H) were assessed by quantitative reverse transcriptase PCR in GCA artery samples obtained from patients with GCA and controls. Data are expressed as individual data points (mean). ATLOs, artery tertiary lymphoid organs; HC, healthy controls. 
presence of giant cells are not sufficient to determine the progression of lymphoid organisation. We also evaluated whether the presence of ATLOs was associated with atherosclerotic lesions. Presence of ATLOs was associated with the presence of atheromatous plaques in the arterial tunica intima in only 2 of 30 patients (figure 1O). Finally, since ATLO formation appears to be related with ageing, the occurrence of ATLOs was correlated with the age of patients with GCA. ATLOs were similarly observed in patients with GCA independently by the age (figure 1P). Collectively these data indicated that ATLOs formation occurs in a significant proportion of GCA arteries independently by the intensity of tissue inflammation, age and the presence of atherosclerosis.

\section{Differential expression of chemokines and chemokine receptors and cytokines involved in lymphoneogenesis in GCA arteries}

Since the occurrence of ATLOs in patients with GCA, we next evaluated the expression levels of different chemokines and chemokine receptors and cytokines involved in lymphoneogenesis: CXCL13, CXCR5, CCL21, CCR7, lymphotoxin (LT)- $\beta$, BAFF, a proliferation-inducing ligand (APRIL), IL-7 and IL-17. As shown in figure 3, significant overexpression of CXCL13 (figure 3A), CXCR5 (figure 3B), lymphotoxin (LT)- $\beta$ (figure 3C), BAFF (figure $3 \mathrm{D}$ ), a proliferation-inducing ligand (APRIL) (figure $3 \mathrm{E}$ ), IL-7 (figure 3F), IL-7R (figure 3G) and IL-17 (figure 3H) was observed in ATLOs $^{+}$compared with ATLOs ${ }^{-}$arteries and controls. IL-17 and IL-7 expression was significantly correlated with the levels of CXCL13 only in ATLO-positive arteries (IL-7: r2=0.36, $\mathrm{p}=0.0001$; IL-17: $\mathrm{r} 2=0.13, \mathrm{p}=0.02)$. Conversely, no significant increase of CCL21 and its receptor CCR7 was observed in all the inflamed arteries independently by the presence of ATLOs (data not shown). The expression of CXCL13, BAFF and lymphotoxin (LT) $\beta$ was next studied by immunohistochemistry (IHC). The presence of ATLOs in GCA arteries was associated with CXCL13 (figure 4A-D), BAFF (figure 4E-H) and lymphotoxin (LT)- $\beta$ expression (figure 4I-L). In particular, immunohistochemistry (IHC) staining showed a strong expression of CXCL13 and BAFF within infiltrating cells, vascular smooth muscle cells and HEVs. Interestingly, lymphotoxin (LT)- $\beta$ was significantly overexpressed by lymphoid cells in both arteries with (figure 4I) and without (figure 4J) ATLOs. Since the localisation of ATLOs in the media layer and the strong expression of CXCL13 and BAFF in the context of vascular smooth muscle cells, we next evaluated their expression in primary cultures of myofibroblasts obtained from temporal arteries of five GCA and three control arteries. The expression of CXCL13, BAFF and lymphotoxin (LT)- $\beta$ in myofibroblasts was assessed by RT-PCR and demonstrated in GCA a significant overexpression of CXCL13 (see online supplementary figure S1A), CXCR5 (see online supplementary figure S1B), BAFF (see online supplementary figure S1C) but also of lymphotoxin
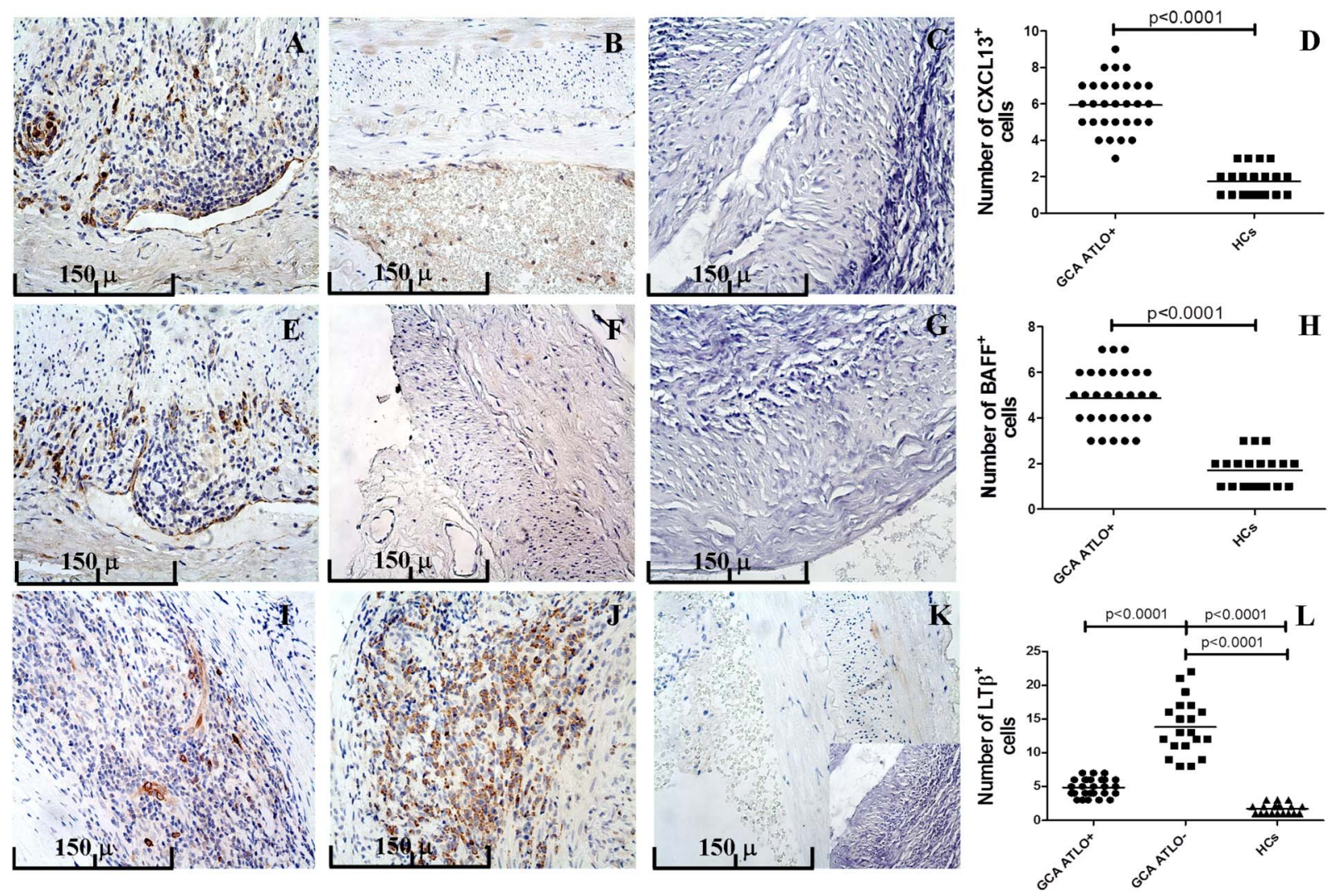

Figure 4 CXCL13, B cell activating factor (BAFF) and lymphotoxin (LT)- $\beta$ expression in giant cell arteritis (GCA) temporal arteries. Representative microphotographs showing CXCL13 expression in arteries obtained from patients with GCA with artery tertiary lymphoid organs (ATLOs) (A) and normal controls (B). (C) Representative image showing GCA arteries stained with isotype control antibody. (D) Semiquantitative evaluation of CXCL13-positive cells. Representative microphotographs showing BAFF expression in arteries obtained from GCA patients with ATLOs (E) and normal controls (F). (G) Representative image showing GCA arteries stained with isotype control antibody. (H) Semiquantitative evaluation of BAFF-positive cells. Representative microphotographs showing lymphotoxin (LT)- $\beta$ expression in arteries obtained from patients with GCA with ATLOs (I), without ATLO $(\mathrm{J})$ and control $(\mathrm{K})$. Inset in $(\mathrm{K})$ shows GCA arteries stained with isotype control antibody. (L) Semiquantitative evaluation of lymphotoxin (LT)- $\beta$-positive cells. Data are expressed as individual data points (mean). 
(LT)- $\beta$ (see online supplementary figure S1D). These data seem to indicate that stromal cells of GCA arteries are equipped with pro-inflammatory chemokines involved in lymphoneogenesis.

\section{In vitro stimulation of primary cultures from temporal} arteries with TLR agonists and cytokines modulates CXCL13, BAFF, a proliferation-inducing ligand (APRIL) and CCL21 production

Since the increased expression in GCA myofibroblasts of chemokines involved in lymphoneogenesis, we next evaluated whether the expression of CXCL13, BAFF, a proliferation-inducing ligand (APRIL) and CCL21 may be modulated by Toll-like receptor (TLR) agonists and cytokines. IL-17, IL-6, poly-IC and LPS stimulation of primary cultures of myofibroblasts resulted in a significant upregulation of CXCL13 (figure 5A) and BAFF (figure 5B). BAFF was significantly upregulated also by IFN $\gamma$, whereas a proliferation-inducing ligand (APRIL) overexpression was induced only after IL-6 stimulation (figure 5C). Finally, CCL2 1 was upregulated by IL-1 $\beta$, IFN $\gamma$, IL-17 and IL- 6 but not by TLR agonists (figure 5D). These findings demonstrate a fine and differential regulation of different chemokines in myofibroblasts of GCA.

\section{Lymphatic conduits are present in GCA arteries with ATLOs and connect ATLOs with muscular layer}

Lymphatic conduits are the main way used by antigens to enter in lymphoid structures, and their presence has been demonstrated in atherosclerotic arteries forming a reticular meshwork of fibres enfolded by podoplanin-expressing fibroblastic reticular cells. ${ }^{3}$ In order to evaluate the presence of lymphatic conduits in GCA arteries, staining with 8.1.1 (podoplanin/gp38) was performed. Intense podoplanin staining was consistently detected in the areas where ATLOs were present (figures 2 and 6A) as well as on lymphatic vessels outside the germinal centre (figure 6B), but not in control arteries.

\section{FDC perivascular precursors are expanded in GCA arteries}

FDCs are essential in the development and maintenance of lymphoid follicles. ${ }^{18}$ It has been recently demonstrated that FDCs derive from ubiquitous perivascular precursors and that they are dependent for their proliferation by lymphotoxin (LT)- $\alpha \beta$-producing lymphoid tissue inducer (lymphotoxin (LT)i) cells. ${ }^{19}$ As shown in figures 2 and 7, FDC precursors, identified as $\mathrm{CXCL}_{13}{ }^{+} / \mathrm{NG}^{+} /$platelet-derived growth factor receptors (PDGFR) $\mathrm{b}^{+}$cells, were present in the majority of inflamed arteries being the highest number observed in the presence of ATLO.

\section{DISCUSSION}

In chronic inflammatory diseases, primary $\mathrm{T}$ cell responses are thought to be initiated in secondary lymphoid organ (SLOs) and in TLOs, unencapsulated lymphoid aggregates occurring postnatally at undetermined sites in response to non-resolving

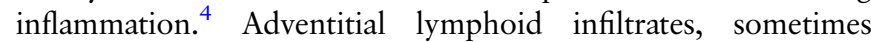
aggregated in lymphoid follicles (so-called ATLOs), have been demonstrated to increase with age and to be associated with the presence of atherosclerosis. $^{3}$

GCA is the prototype of large vessel vasculitis and has been mainly considered to be driven by a cell-mediated reaction involving activated macrophages, multinucleate giant cells and $\mathrm{T}$
A

CXCL13

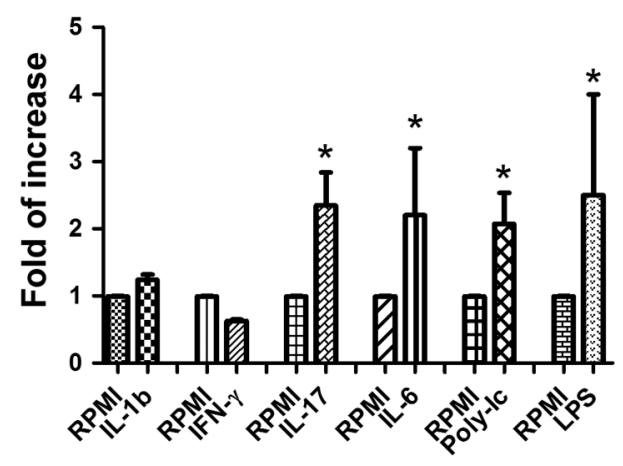

C

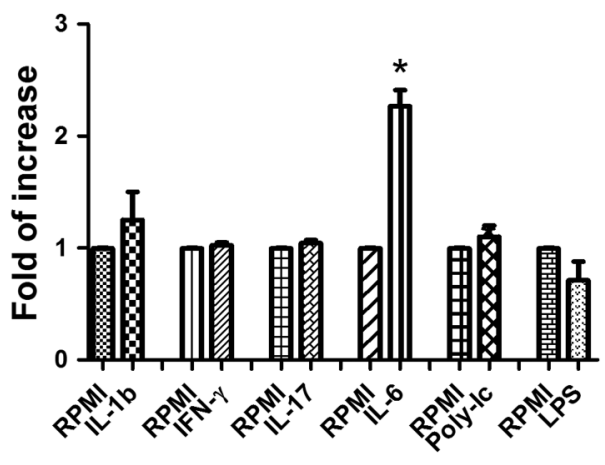

B

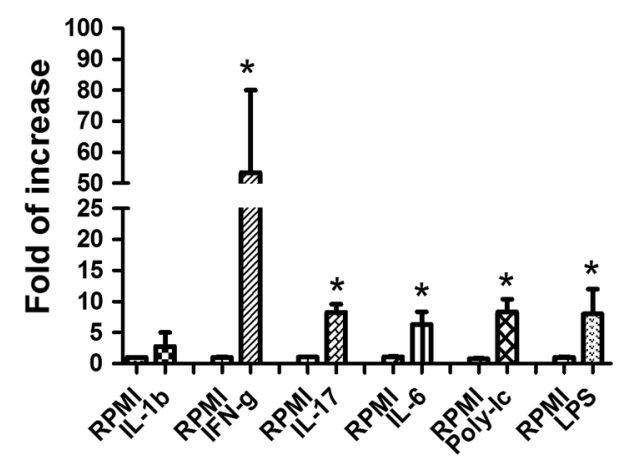

D

CCL21

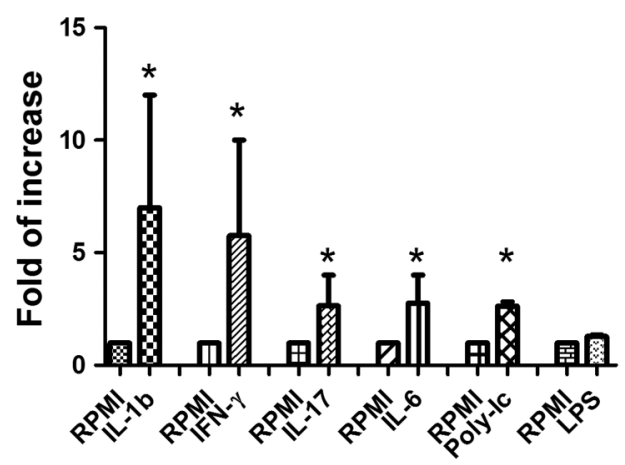

Figure 5 Chemokine modulation in primary cultures from temporal arteries. Myofibroblast-like cultures were obtained from temporal arteries of patients with giant cell arteritis and controls and cultured in the presence of different stimuli. Modulation of chemokines expression was assessed by reverse transcriptase PCR. (A) CXCL13 modulation by different pro-inflammatory cytokines and Toll-like receptor (TLR) agonists. (B) B cell activating factor (BAFF) modulation by different pro-inflammatory cytokines and TLR agonists. (C) a proliferation-inducing ligand (APRIL) modulation by different pro-inflammatory cytokines and TLR agonists. (D) CCL21 modulation by different pro-inflammatory cytokines and TLR agonists. IL, interleukin; LPS, lipopolysaccharide; RPMI, Roswell Park Memorial Institute medium. 


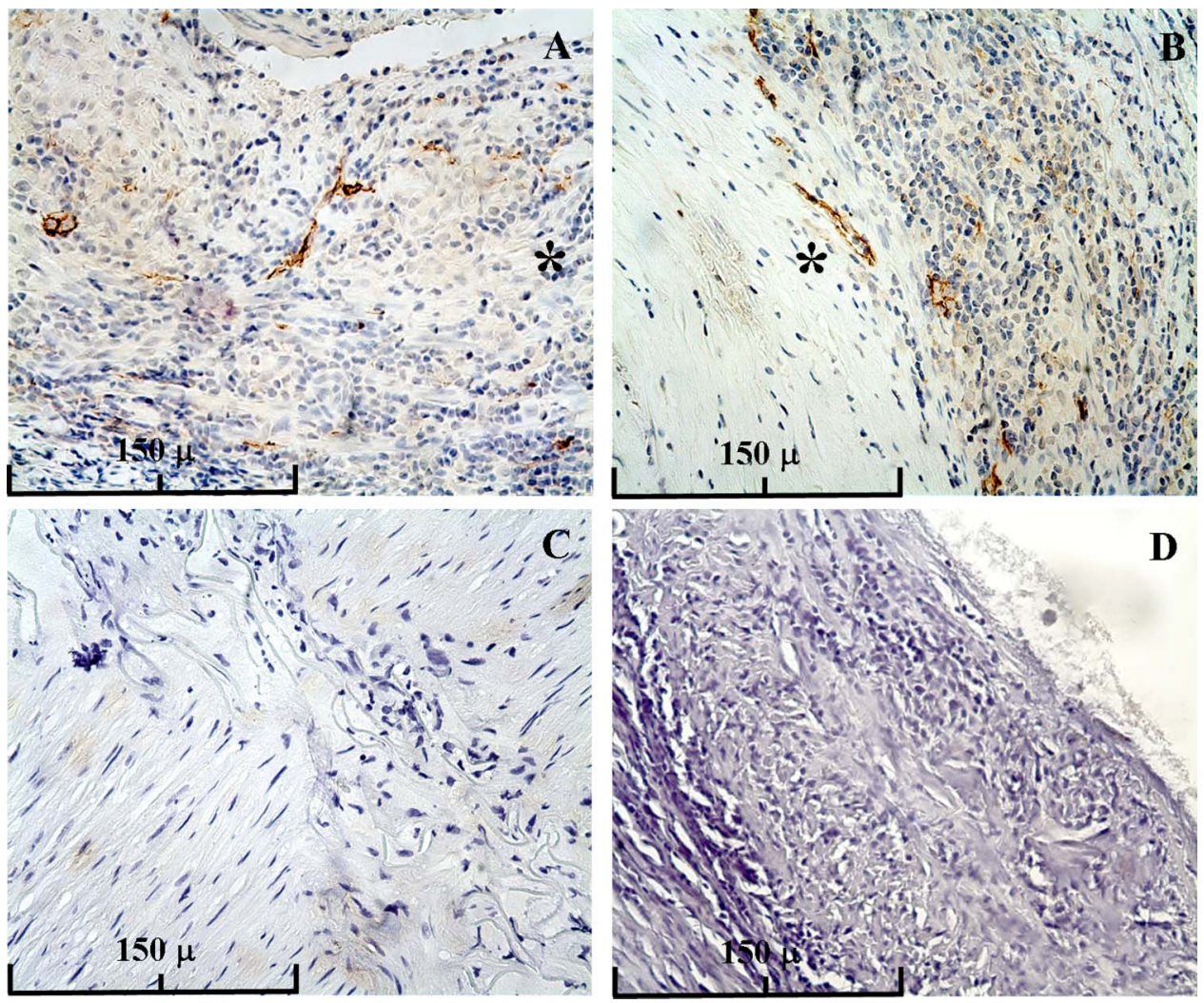

Figure 6 Ectopic lymphoid conduits are present in giant cell arteritis (GCA) temporal arteries. Representative microphotographs showing podoplanin ${ }^{+}$lymphoid vessels in GCA arteries in the context of artery tertiary lymphoid organ (ATLO) structures (A) and outside of ATLOs (B). (C) Representative microphotographs showing podoplanin staining in normal arteries. (D) Representative microphotographs showing GCA arteries stained with isotype control antibody.

lymphocytes. ${ }^{9}$ The presence of ectopic lymphoid organs has been demonstrated in chronic periaortitis ${ }^{12}$ but not in GCA and the microanatomic association between expression of cytokines and chemokines involved in lymphoneogenesis and the acquisition of lymphoid features in inflamed arteries not yet studied.

In this study, we demonstrated for the first time, to our knowledge, that distinct ATLOs structures, including placed B cell aggregates with a FDC network, loosely surrounded by $\mathrm{T}$ cells, and the extensive formation of HEV, occur in a significant proportion of patients with GCA (an image that summarises the structure of ATLOs in GCA arteries is shown in figure 2). HEVs are specialised postcapillary venous characterised by almost cuboidal endothelial cells that support high levels of lymphocyte extravasation from the blood. HEV-like vessels have been demonstrated in chronically inflamed non-lymphoid tissues, and their differentiation appears to be under the control of the local tissue environment. ${ }^{20}$ In GCA, neo-angiogenesis occurs in the media layer where HEVs are mainly observed, indicating that cytokines/chemokines released by vascular smooth muscle cells (VSMCs) may be essential in determining HEV differentiation. The spatial distribution of HEV in GCA may be responsible for the peculiar location of GCA lymphoid aggregates that, differently from 'classic' ATLOs, were mainly observed in the media layer of inflamed temporal arteries. ATLOs were not associated with the age of patients and/or with the occurrence of atherosclerotic lesions, and were also independent by the degree of arterial inflammation. In addition, newly formed lymphatic conduits establish a network connecting ATLOs to the medial vascular smooth muscle cells (VSMC). This conduit network has been demonstrated to serve as channels for the transport of soluble antigen and chemokines/cytokines between the activated vascular smooth muscle cells (VSMCs) and ATLOs. ${ }^{19} 2122$

FDCs are essential in engaging B cells in the SLOs and TLOs. FDCs have been demonstrated to emerge from ubiquitous perivascular precursors also in non-lymphoid organs. ${ }^{23}$ FDCs generated de novo in TLO seem to arise from these progenitor cells when exposed to the appropriate environment. Herein we demonstrated that perivascular FDC precursors are present in the media layer of GCA arteries with ATLOs, indicating that a specific chemokine and cytokine environment may induce their differentiation in mature FDC and the appearance of tertiary lymphoid structures.

CXCL13 and CCL21, produced by vascular smooth cells, have been demonstrated to be instrumental in maintaining FDC networks in mouse arteries, and IL-7/IL-17 axis has been also demonstrated to be required for the organisation of ectopic lymphoid structures. ${ }^{3}{ }^{24} 25$ In particular, CXCL13-attracted B cells, which are home to the follicles, are the source of lymphotoxin (LT)- $\alpha 1 \beta 2,{ }^{25}$ which is critical for the generation and maintenance of established follicles. Analysis of tissue expression of cytokines, chemokines and their receptors in ATLOs-positive GCA arteries demonstrated a clear increased expression of IL-17 and IL-7, correlated with that of CXCL13, and of their receptor IL-7R and CXCR5. Interestingly, BAFF, a proliferationinducing ligand (APRIL) and lymphotoxin (LT)- $\beta$ were also upregulated, whereas no significant overexpression of CCL21 and of its receptor CCR7 was observed. lymphotoxin (LT)- $\beta$ was essentially expressed among infiltrating lymphoid cells. Conversely, CXCL13 and BAFF were predominantly expressed among vascular smooth muscle cells (VSMCs) and endothelial 

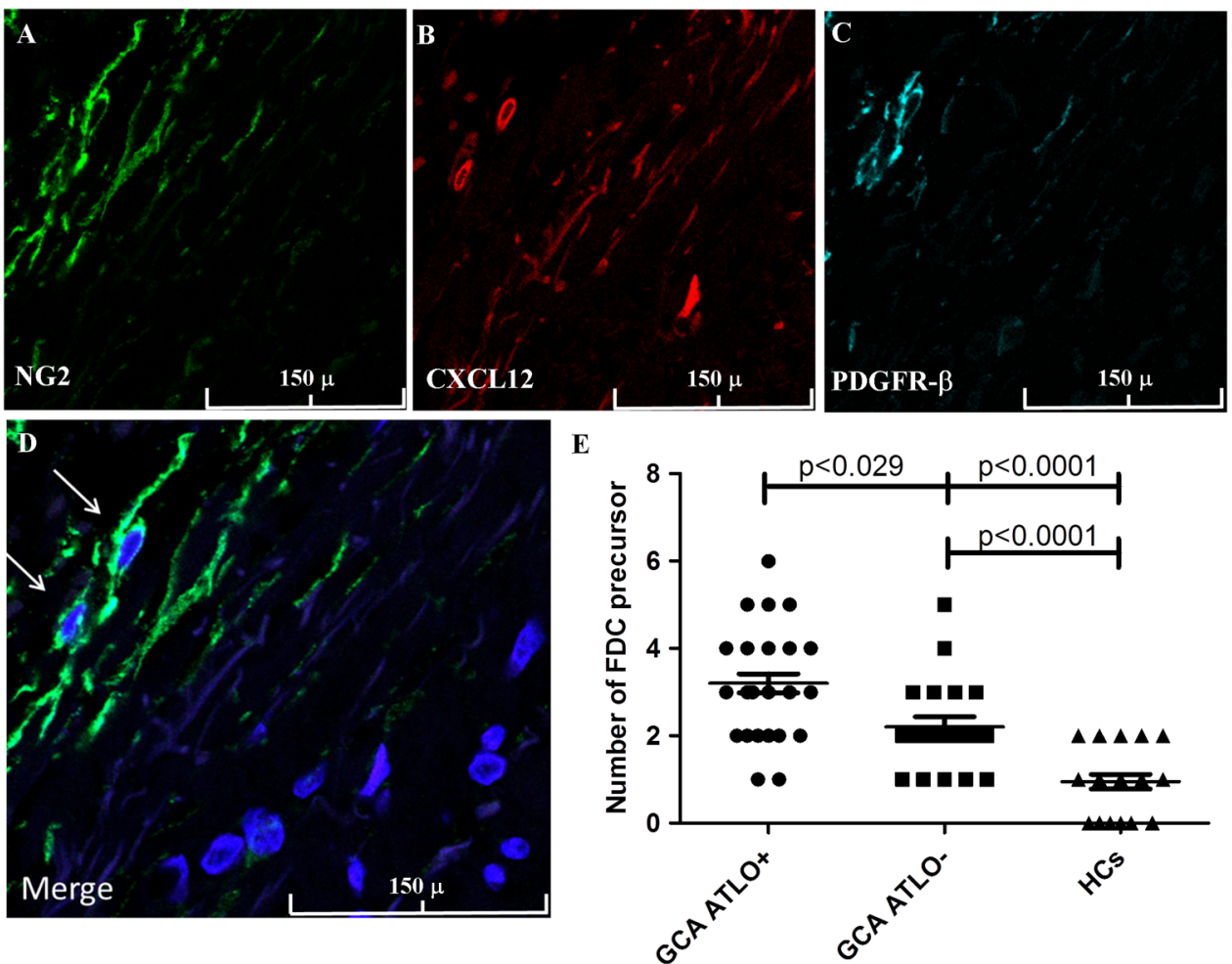

Figure 7 Follicular dendritic cell (FDC) precursors in giant cell arteritis (GCA). (A) Single staining for NG2; (B) single staining for CXCL12; (C) single staining for platelet-derived growth factor receptors (PDGFR)- $\beta$; (D) merge triple staining for NG2, CXCL12 and platelet-derived growth factor receptors (PDGFR)- $\beta$. (E) quantification of FDC precursors in GCA and normal arteries.

cells of HEV. Microvascular endothelial cells have been demonstrated to produce BAFF and a proliferation-inducing ligand (APRIL) constituting a key component of the neoplastic stroma by cross-talking with neoplastic B cells. ${ }^{26}$ Our findings indicate that HEV cells may be also essential in establishing an intimate cross-talk with artery infiltrating B cells through the production of B-cell-activating chemokines.

Immune-stromal communications between T cells and myointimal cells have been previously demonstrated in GCA. ${ }^{9}$ In this work, we provide the evidence that primary human temporal artery-derived myointimal cells may also interact with B cells via the production of B-cell-attracting chemokines in GCA. We demonstrated that primary cultures of myofibroblasts obtained from temporal arteries constitutively express large amounts of CXCL13 and BAFF, and that expression of CXCL13, BAFF, a proliferation-inducing ligand (APRIL) and CCL21 is differentially regulated by TLR agonists and cytokines. TLR3 and TLR4 stimulation in fact induced in such primary cultures a strong and rapid upregulation of both BAFF and CXCL13 but not of CCL21 and a proliferation-inducing ligand (APRIL). Conversely, stimulation with different pro-inflammatory cytokines such as IL-1 $\beta$, IL-6, IL-17 and IFN $\gamma$, involved in the pathogenesis of GCA, differentially resulted in the significant upregulation of BAFF, CXCL13, CCL21 and in a less manner of a proliferationinducing ligand (APRIL). TLR3 and TLR4 are members of the TLR family recognising pathogen-associated molecular patterns expressed on infectious agents, and mediating the production of cytokines of innate immunity. ${ }^{27}$ Vascular smooth muscle cells have been demonstrated to express TLR $3^{28}$ and TLR $4,{ }^{29}$ and their stimulation, potentially triggered by infective agents, might mediate the early release of CXCL13 and BAFF, thus attracting lymphotoxin (LT)i cells and inducing their initial clustering. ${ }^{30}$
In summary, in this study we report that (1) ATLOs structures occur in GCA arteries; (2) IL-7 and IL-17 are associated with the presence of ATLOs structures; (3) ectopic expression of CXCL13, BAFF and lymphotoxin (LT)- $\beta$ is associated with the presence of ATLOs; (4) BAFF and CXCL13 are induced by TLR3 and TLR4 ligands; and (5) BAFF and CXCL13 are induced by pro-inflammatory cytokines. Further studies are required to better clarify the clinical relevance of ATLOs in GCA.

\section{Author affiliations}

${ }^{1}$ Dipartimento Biomedico di Medicina Interna e Specialistica, Sezione di Reumatologia, Università degli Studi di Palermo, Palermo, Italy

2Dipartimento di Oncoematologia, Sezione di Anatomia Patologica, Azienda Ospedaliera Ospedali riuniti Villa Sofia Cervello, Palermo, Italy

${ }^{3}$ Dipartimento di Emergenze, Urgenze e Neuroscienze Cliniche, Università di Palermo, Palermo, Italy

${ }^{4}$ Dipartimento di Biopatologia e Biotecnologie Mediche e Forensi, Università di Palermo, Palermo, Italy

${ }^{5}$ Clinical Immunology, Allergy and Advanced Biotechnologies Unit, Arcispedale Santa Maria Nuova, IRCCS, Reggio Emilia, Italy

${ }^{6}$ Pathology Unit, Arcispedale Santa Maria Nuova, IRCCS, Reggio Emilia, Italy ${ }^{7}$ Unità operativa di Reumatologia, Arcispedale S. Maria Nuova-IRCCS, Reggio Emilia, Italy

Acknowledgements The authors thank Dr Francesca Raiata (Sezione di Anatomia Patologica, Azienda Ospedaliera Ospedali riuniti Villa Sofia Cervello, Palermo, Italy) for her technical support in immunohistochemical experiments and Dr Maria-Cinta Cid (Hospital Clinic de Barcelona, Spain) for having trained Dr Stefania Croci in cultures from temporal arteries. They also thank Dr Angelo Ferrante (Rheumatology, University of Palermo) for his help in drawing figure 2.

Contributors CS and GT shared co-senior authorship. Study design: FC, AR, CS and GT. Acquisition of data: RM, RA, SC, GG, AC, SR, AC and DGI. Analysis and interpretation of data: FC, AR, CS, GT, SC, RM, RA, SC, GG, SR and DGI. Manuscript preparation: FC, CS and GT. Statistical analysis: FC and AC. Overall supervision: GT, CS. 
Funding This study was in part supported by a grant of Ministero dell'Istruzione, dell'Università e della ricerca Scientifica from Italy

Competing interests None declared.

Patient consent Obtained.

Ethics approval University of Palermo.

Provenance and peer review Not commissioned; externally peer reviewed.

\section{REFERENCES}

1 Dal Canto AJ, Swanson PE, O'Guin AK, et al. IFN-gamma action in the media of the great elastic arteries, a novel immunoprivileged site. J Clin Invest 2001;107:R15-22.

2 Hu D, Mohanta SK, Yin C, et al. Artery tertiary lymphoid organs control aorta immunity and protect against atherosclerosis via vascular smooth muscle cell lymphotoxin $\beta$ receptors. Immunity 2015;42:1100-15.

3 Gräbner $\mathrm{R}$, Lötzer $\mathrm{K}$, Döpping $\mathrm{S}$, et al. Lymphotoxin $\beta$ receptor signaling promotes tertiary lymphoid organogenesis in the aorta adventitia of aged ApoE-/- mice. J Exp Med 2009;206:233-48.

4 Pitzalis $\mathrm{C}$, Jones GW, Bombardieri $\mathrm{M}$, et al. Ectopic lymphoid-like structures in infection, cancer and autoimmunity. Nat Rev Immunol 2014;14:447-62.

5 Amft N, Curnow SJ, Scheel-Toellner D, et al. Ectopic expression of the B cell-attracting chemokine BCA-1 (CXCL13) on endothelial cells and within lymphoid follicles contributes to the establishment of germinal center-like structures in Sjögren's syndrome. Arthritis Rheum 2001;44:2633-41.

6 Timmer TC, Baltus B, Vondenhoff M, et al. Inflammation and ectopic lymphoid structures in rheumatoid arthritis synovial tissues dissected by genomics technology: identification of the interleukin-7 signaling pathway in tissues with lymphoid neogenesis. Arthritis Rheum 2007;56:2492-502.

7 Liu X, Leung S, Wang C, et al. Crucial role of interleukin-7 in Thelper type 17 survival and expansion in autoimmune disease. Nat Med 2010;16:191-7

8 Salvarani C, Pipitone N, Versari A, et al. Clinical features of polymyalgia rheumatica and giant cell arteritis. Nat Rev Rheumatol 2012;8:509-21.

9 Weyand CM, Goronzy JJ. Immune mechanisms in medium and large-vessel vasculitis. Nat Rev Rheumatol 2013;9:731-40.

10 Cid MC, Campo E, Ercilla G, et al. Immunohistochemical analysis of lymphoid and macrophage cell subsets and their immunologic activation markers in temporal arteritis. Influence of corticosteroid treatment. Arthritis Rheumatol 1989;32:884-93.

11 van der Geest KS, Abdulahad WH, Chalan P, et al. Disturbed B cell homeostasis in newly diagnosed giant cell arteritis and polymyalgia rheumatica. Arthritis Rheumatol 2014;66:1927-38.

12 Vaglio A, Pipitone N, Salvarani C. Chronic periaortitis: a large-vessel vasculitis? Curr Opin Rheumatol 2011;23:1-6.

13 Hunder GG, Bloch DA, Michel BA, et al. The American College of Rheumatology 1990 criteria for the classification of giant cell arteritis. Arthritis Rheum 1990;33:1122-8.

14 Hernández-Rodríguez J, Segarra M, Vilardell C, et al. Elevated production of interleukin-6 is associated with a lower incidence of disease-related ischemic events in patients with giant-cell arteritis: angiogenic activity of interleukin- 6 as a potential protective mechanism. Circulation 2003;107:2428-34.

15 Ciccia F, Rizzo A, Guggino G, et al. Difference in the expression of IL-9 and IL-17 correlates with different histological pattern of vascular wall injury in giant cell arteritis. Rheumatology (Oxford) 2015;54:1596-604.

16 Bombardieri $\mathrm{M}$, Barone $\mathrm{F}$, Pittoni $\mathrm{V}$, et al. Increased circulating levels and salivary gland expression of interleukin-18 in patients with Sjögren's syndrome: relationship with autoantibody production and lymphoid organization of the periductal inflammatory infiltrate. Arthritis Res Ther 2004;6:R447-56.

17 Lozano E, Segarra M, García-Martínez A, et al. Imatinib mesylate inhibits in vitro and ex vivo biological responses related to vascular occlusion in giant cell arteritis. Ann Rheum Dis 2008:67:1581-8.

18 Hutchinson E. Lymphoid follicles: FDCs — ubiquitous and ready for action. Nat Rev Immunol 2012;12:617.

19 Nolte MA, Beliën JA, Schadee-Eestermans I, et al. A conduit system distributes chemokines and small blood-borne molecules through the splenic White pulp. J Exp Med 2003;198:505-12.

20 Hayasaka H, Taniguchi K, Fukai S, et al. Neogenesis and development of the high endothelial venules that mediate lymphocyte trafficking. Cancer $\mathrm{SCi}$ 2010;101:2302-8.

21 Sixt M, Kanazawa N, Selg M, et al. The conduit system transports soluble antigens from the afferent lymph to resident dendritic cells in the T cell area of the lymph node. Immunity 2005;22:19-29.

22 Itano AA, Jenkins MK. Antigen presentation to naive CD4T cells in the lymph node. Nat Immunol 2003;4:733-9.

23 Krautler NJ, Kana V, Kranich J, et al. Follicular dendritic cells emerge from ubiquitous perivascular precursors. Cell 2012;150:194-206.

24 Grogan JL, Ouyang W. A role for Th17 cells in the regulation of tertiary lymphoid follicles. Eur J Immunol 2012;42:2255-62.

25 Litsiou E, Semitekolou M, Galani IE, et al. CXCL13 production in B cells via Toll-like receptor/lymphotoxin receptor signaling is involved in lymphoid neogenesis in chronic obstructive pulmonary disease. Am J Respir Crit Care Med 2013;187:1194-202.

26 Cols M, Barra CM, He B, et al. Stromal endothelial cells establish a bidirectional crosstalk with chronic lymphocytic leukemia cells through the TNF-related factors BAFF, a proliferation-inducing ligand (APRIL), and CD40L. J Immunol 2012;188:6071-83.

27 Beutler BA. TLRs and innate immunity. Blood 2009;113:1399-407.

28 Yang $\mathrm{X}$, Murthy $\mathrm{V}$, Schultz $\mathrm{K}$, et al. Toll-like receptor 3 signaling evokes a proinflammatory and proliferative phenotype in human vascular smooth muscle cells. Am J Physiol Heart Circ Physiol 2006;291:H2334-43.

29 Sasu S, LaVerda D, Qureshi N, et al. Chlamydia pneumoniae and chlamydial heat shock protein 60 stimulate proliferation of human vascular smooth muscle cells via toll-like receptor 4 and p44/p42 mitogen-activated protein kinase activation. Circ Res 2001;89:244-50.

30 van de Pavert SA, Olivier BJ, Goverse G, et al. Chemokine CXCL13 is essential for lymph node initiation and is induced by retinoic acid and neuronal stimulation. Nat Immunol 2009;10:1193-9. 


\section{ARD}

Ectopic expression of CXCL13, BAFF, APRIL and $L T-\beta$ is associated with artery tertiary lymphoid organs in giant cell arteritis

Francesco Ciccia, Aroldo Rizzo, Rosario Maugeri, Riccardo Alessandro, Stefania Croci, Giuliana Guggino, Alberto Cavazza, Stefania Raimondo, Alessandra Cannizzaro, Domenico Gerardo lacopino, Carlo Salvarani and Giovanni Triolo

Ann Rheum Dis published online April 20, 2016

Updated information and services can be found at:

http://ard.bmj.com/content/early/2016/04/20/annrheumdis-2016-2092 17

References

\section{Email alerting} service

\section{These include:}

This article cites 30 articles, 9 of which you can access for free at: http://ard.bmj.com/content/early/2016/04/20/annrheumdis-2016-2092 17\#BIBL

Receive free email alerts when new articles cite this article. Sign up in the box at the top right corner of the online article.

Topic Articles on similar topics can be found in the following collections Collections

\section{Notes}

To request permissions go to:

http://group.bmj.com/group/rights-licensing/permissions

To order reprints go to:

http://journals.bmj.com/cgi/reprintform

To subscribe to BMJ go to:

http://group.bmj.com/subscribe/ 